\title{
OF THE AIR
}

Edmun B. Richmond, Editor

\section{Short Wave Receiving Edmun Richmond}

With this issue of NALLDJ: Technology and Mediated Instruction, a new column is hereby inaugurated to focus on the effective use of foreign language materials broadcast over the airwaves via radio or television. An outgrowth of the former, Language By Radio Interest Group (LBRIG), the column will report on current broadcasting stations, teaching materials, receivers, antennas, and techniques for the reception of the desired signals.

\section{Reception}

Initially, the reception of amateur radio signals will be discussed. Such transmissions are available around the clock in almost every imaginable language and can add an air of realistic conversation to any foreign language class.

The optimal frequencies to receive international amateur radio conversations are the 20,15 and 10 meter bands which correspond to the following frequencies:

$$
\begin{aligned}
& 20 \text { meters }=14.100-14.350 \mathrm{mHz} \\
& 15 \text { meters }=21.000-21.450 \mathrm{mHz} \\
& 10 \text { meters }=28.000-29.700 \mathrm{mHz}
\end{aligned}
$$

Generally the $\mathbf{2 0}$ meter band will be the most fruitful since it is open to most of the world twenty-four hours a day with North and South American stations continuously transmitting. European stations will usually be heard on $\mathbf{2 0}$ meters in the late afternoon and throughout the evening and night hours. Reception also improves during the cold winter months.

The 15 and 10 meter bands are usually "daylight bands" and open up at local sunrise. It must be noted that signal propagation and reception are subject to many variables including sunspots, weather, and even the outside air temperature. Thus, a frequency good on Monday might be totally unusable on Tuesday.

Since most amateur radio stations transmit and receive on the same frequency, it will often be possible to hear both ends of a conversation. However, reception of thest signals requires a receiver equipped to receive single sideband (SSB) transmissions. On the three international amateur bands, most amateurs transmit on upper sideband, marked USB on the receiver. 


\title{
Callsigns
}

Identification of the country of origin is made easy by the fact that each country in the world has a unique set of callsigns, all of which are listed in the Radio Amateur Callbook.

\section{Equipment}

Teachers or other individuals interested in acquiring a shortwave receiver should specify a general purpose receiver with a Bandspread and Single Sideband capability. Such a receiver will permit the reception not only of amateur radio signals, but also the international shortwave frequencies.

Future editions of the Joumal will include articles and bibliographies on the selection and use of shortwave receivers. In addition, the Joumal welcomes equipment descriptions along with comments and questions from the readers. Please address all letters on shortwave broadcasts to Prof. Edmun Richmond, Modern Language Dept., Georgia Institute of Technology, Atlanta, GA 30332.

\section{NALLD NOTE from the Editor-inChief}

There is growing interest among both professional and amateur electronics buffs in the reception of signals (particularly in foreign languages) from the many geostationary earth satellites parked in orbits over the equator. In the November 1979 issue of 73 Magazines for Radio Amateurs, Mr. Robert Cooper, W5KHT, had a very interesting article entitled "The tSaellite TV Primer" which provided an excellent insight into this new and exciting field.

For additional information contact:

\author{
Mr. Robert Cooper \\ Satellite Television Technology \\ POB G \\ Arcadia, OK 73007 \\ 405-396-2574
}

